\title{
ENCEFALOPATIA DIABÉTICA E DEPRESSÃO: DANO OXIDATIVO NO CÉREBRO
}

\author{
DIABETIC ENCEPHALOPATHY AND DEPRESSION: \\ BRAIN OXIDATIVE DAMAGE
}

\author{
Simone Mattos Louzada ${ }^{1}$, Carmen Regla Vargas ${ }^{1,2,3}$
}

Clin Biomed Res. 2015;35(4):184-195

1 Curso de Especialização em Análises Clínicas, Universidade Federal do Rio Grande do Sul (UFRGS). Porto Alegre, RS, Brasil.

2 Programa de Pós-graduação em Ciências Farmacêuticas, Universidade Federal do Rio Grande do Sul (UFRGS) Porto Alegre, RS, Brasil.

3 Serviço de Genética Médica, Hospital de Clínicas de Porto Alegre. Porto Alegre, RS, Brasil.

Autor correspondente: Carmen Regla Vargas E-mail: crvargas@hcpa.edu.br Serviço de Genética Médica, Hospital de Clínicas de Porto Alegre

Rua Ramiro Barcelos, 2350. 90035-903, Porto Alegre, RS, Brasil.

\section{RESUMO}

O diabetes é um distúrbio complexo e heterogêneo caracterizado por hiperglicemia resultante de defeitos na secreção e ação da insulina. Tem sido reconhecido que, além do comprometimento de órgãos como rins, olhos, fígado e coração, o sistema nervoso central é suscetível aos efeitos deletérios da hiperglicemia em longo prazo. A encefalopatia diabética representa uma das complicações do diabetes, na qual os danos são caracterizados por alterações do funcionamento cognitivo, modificações estruturais e neurofisiológicas no cérebro. Existe uma associação bem reconhecida entre a depressão e o diabetes, uma vez que a prevalência de depressão é maior na população diabética comparada com a população geral. Porém, os mecanismos atribuídos a essa relação ainda estão em fase de investigação. O estresse oxidativo desempenha um papel importante nas complicações do diabetes e pode ser um mecanismo biológico envolvido na relação entre a depressão e o diabetes, relacionado à encefalopatia diabética. Neste artigo de revisão, apresentamos uma visão geral dos principais conceitos relacionados ao assunto, bem como dos dados clínicos e experimentais que suportam a relação entre o dano oxidativo no cérebro e a depressão relacionada com encefalopatia diabética.

Palavras-chave: Diabetes; depressão; encefalopatia diabética; cérebro; estresse oxidativo; antioxidantes

\section{ABSTRACT}

Diabetes is a complex and heterogeneous disorder characterized by hyperglycemia resulting from defects in the secretion and action of insulin. It has been recognized that, in addition to the involvement of organs such as kidney, eye, liver, and heart, the central nervous system is susceptible to the deleterious effects of hyperglycemia in the long term. Diabetic encephalopathy is one of the complications of diabetes, in which the damage is characterized by changes in cognitive functioning, structural and neurophysiologic changes in the brain. There is a well-known association between depression and diabetes, since the prevalence of depression is higher in the diabetic population compared to the general population. However, the mechanisms assigned to this relationship are still under investigation. Oxidative stress plays an important role in the complications of diabetes and can be a biological mechanism involved in the relation between depression and diabetes related to diabetic encephalopathy. This review article is an overview of key concepts related to the subject, as well as of the clinical and experimental data supporting the relationship between oxidative damage in the brain and depression related to diabetic encephalopathy.

Keywords: Diabetes; depression; diabetic encephalopathy; brain; oxidative stress; antioxidants 
O diabetes é uma desordem complexa e heterogênea do metabolismo, em que ocorre hiperglicemia crônica. Nessa patologia, ocorre um prejuízo na secreção e na ação do hormônio insulina, o principal regulador da homeostase glicêmica ${ }^{1}$. Vários processos patogênicos estão envolvidos no desenvolvimento do diabetes, os quais vão desde a destruição autoimune das células- $\beta$ do pâncreas, com consequente deficiência de insulina - caracterizando o diabetes tipo 1 -, ou anomalias decorrentes de um defeito progressivo na secreção de insulina que resultam em resistência à ação da insulina - caracterizando o diabetes tipo $2^{2}$. Globalmente, estima-se que 387 milhões de pessoas sofram de diabetes (prevalência de 8,3\%); no Brasil, a estimativa é de 11.934 milhões $^{3}$. O diabetes é uma doença de elevada morbidade, pois a sua progressão ocasiona várias complicações que podem afetar órgãos como rins, olhos, coração, vasos sanguíneos e nervos ${ }^{4}$. Além disso, as modificações metabólicas e vasculares gradualmente podem ocasionar danos ao cérebro, resultando em alterações estruturais e eletrofisiológicas e, inclusive, prejuízos à função cognitiva ${ }^{5}$.

Estudos em pacientes diabéticos e em modelo animal experimental de diabetes demonstraram que existe uma relação entre essa doença e deficiências cognitivas $^{6}$. Sabe-se que o sistema nervoso central (SNC) é suscetível às complicações decorrentes do diabetes, provocadas pela hiperglicemia crônica. A encefalopatia diabética é uma dessas complicações, na qual os danos no cérebro são caracterizados por alterações do funcionamento cognitivo, modificações estruturais e neurofisiológicas ${ }^{7}$, podendo se manifestar em pessoas com diabetes tipo 1 e tipo $2^{8}$.

O diabetes está entre as principais causas de incapacidade funcional, assim como a depressão, e ambas as patologias possuem alta incidência na população ${ }^{9}$. A prevalência de depressão é maior em diabéticos quando comparada com a população sem diabetes. Dados estimados indicam que aproximadamente $30 \%$ dos pacientes diabéticos têm sintomas depressivos ${ }^{10}$, representando um grupo propenso a ter inadequada qualidade de vida, gerando maiores gastos com cuidados em saúde e maior risco de mortalidade ${ }^{11}$.

A relação entre depressão e diabetes pode ser atribuída a uma variedade de mecanismos que ainda necessitam de maior entendimento ${ }^{12}$. Nos últimos anos, aumentou o número de evidências de que a depressão seja a expressão clínica da ativação periférica e central de vias imunoinflamatórias e vias de estresse oxidativo, da redução da neurogênese e do aumento de apoptose neuronal ${ }^{13}$. Alguns pesquisadores sugerem que a excessiva produção de espécies reativas de oxigênio (ERO) e o comprometimento das defesas antioxidantes mediadas pela hiperglicemia podem estar envolvidos com o dano tecidual no cérebro de pacientes diabéticos ${ }^{14,15}$. O desequilíbrio entre a produção celular de ERO e os mecanismos antioxidantes que neutralizam essas espécies é denominado estresse oxidativo ${ }^{16}$. Essa alteração do estado redox parece estar associada com o desenvolvimento e a progressão da encefalopatia diabética ${ }^{17,18}$

A encefalopatia diabética pode ser acompanhada de manifestações psiquiátricas ${ }^{19}$. Pouco se sabe a respeito do exato mecanismo dos danos ao SNC relacionados a essa patologia. Recentemente, um estudo verificou que pacientes diabéticos com depressão possuem níveis mais elevados de 8-hidroxi-2'deoxiguanosina (8-OHdG), um indicador de dano oxidativo ao DNA, quando comparados a pacientes diabéticos não portadores de encefalopatia ${ }^{20}$. Dados neuropatológicos post-mortem de córtex pré-frontal de pacientes que apresentavam comportamento depressivo revelaram redução do conteúdo de glutationa (GSH), um antioxidante endógeno, sugerindo que o cérebro desses pacientes seria mais propenso ao dano oxidativo ${ }^{21}$. Indivíduos deprimidos apresentam redução das defesas antioxidantes e aumento de peroxidação lipídica ${ }^{22}$. Resultados semelhantes foram encontrados em estudos com modelo animal experimental de comportamento do tipo depressivo e de diabetes induzido por estreptozotocina (STZ) ${ }^{23}$.

Devido à elevada atividade metabólica, à escassez das defesas antioxidantes e à constituição rica em ácidos graxos poli-insaturados, o cérebro é potencialmente vulnerável ao dano oxidativo ${ }^{24}$. Muitos autores descrevem associações entre o estresse oxidativo e doenças neurológicas como doença de Alzheimer, doença de Parkinson, transtorno bipolar, esquizofrenia, ansiedade e depressão ${ }^{16,25-27}$. Considerando a elevada incidência de diabetes na população e o envolvimento das espécies reativas (ER) no desenvolvimento das complicações diabéticas, como no caso da encefalopatia diabética, é pertinente compreender de que forma o estresse oxidativo pode estar envolvido no elevado risco de desenvolvimento de depressão em diabéticos. Até o momento, não está claro se o estresse oxidativo é causa ou consequência na fisiopatologia do diabetes associado à depressão. Neste artigo de revisão, apresentamos uma visão geral dos principais conceitos relacionados ao assunto, bem como dados clínicos e experimentais que suportam a relação entre o dano oxidativo no cérebro e a depressão relacionada com a encefalopatia diabética. 


\section{ESTRESSE OXIDATIVO E CÉREBRO}

O cérebro é o órgão mais ativo do corpo, devido à sua alta exigência metabólica. Embora compreenda aproximadamente $2 \%$ do peso corporal total no ser humano adulto, esse órgão requer mais de $20 \%$ do suprimento de oxigênio do corpo, o que confere elevada capacidade oxidativa ao permitir amplos substratos metabólicos para geração de $\mathrm{ER}^{25}$. As ERO são produtos de uma série de processos fisiológicos do metabolismo celular oxidativo, incluindo a respiração celular acoplada à fosforilação oxidativa mitocondrial para a formação de adenosina trifosfato (ATP), ações enzimáticas da xantina oxidase e das NADPH oxidases, e o metabolismo do ácido araquidônico pelas lipoxigenases ${ }^{28}$. Na respiração celular, o oxigênio $\left(\mathrm{O}_{2}\right)$ é reduzido à água $\left(\mathrm{H}_{2} \mathrm{O}\right)$ ao receber elétrons de uma só vez pela citocromo oxidase. No entanto, devido à sua conformação eletrônica, o oxigênio tem uma forte tendência a receber um elétron de cada vez, gerando compostos intermediários muito reativos ${ }^{29}$.

Dentre os compostos intermediários formados durante as oxidações biológicas, destacam-se o ânion radical superóxido $\left(\mathrm{O}_{2}^{-}{ }^{-}\right)$, o radical hidroxil $\left(\mathrm{OH}^{*}\right)$ e o peróxido de hidrogênio $\left(\mathrm{H}_{2} \mathrm{O}_{2}\right)$, cuja formação ocorre em aproximadamente $5 \%$ de todo o processo de redução do oxigênio à água. A reação de Fenton, que ocorre da água com íons ferroso ou cúprico, resulta na formação do $\mathrm{OH}^{\circ}$, altamente reativo. Este, por sua vez, também pode ser formado a partir da reação de Haber-Weiss, que ocorre entre $\mathrm{O}_{2}{ }^{-}$e o $\mathrm{OH}_{2} \mathrm{O}_{2}$ na presença de metais. As ERO são altamente oxidantes e podem possuir elétrons desemparelhados, sendo que quando isso ocorre são então denominadas de radicais livres. Além das ERO, existem as espécies reativas do nitrogênio (ERN), como o peroxinitrito (NOO-) e o radical livre óxido nítrico (NO•). Um papel duplo das ER é reconhecido, uma vez que seus efeitos nos organismos vivos podem ser benéficos ou deletérios, dependendo da concentração em que ocorram e das suas funções fisiológicas ${ }^{16}$.

Todas as células que apresentam metabolismo aeróbio estão sujeitas a danos por oxidação, pois a produção das ER ocorre fisiologicamente. No entanto, algumas características tornam o cérebro especialmente sensível às alterações do estado redox. A necessidade de grandes quantidades de ATP para assegurar a neurosecreção e o funcionamento de canais iônicos associados com a propagação de potenciais de ação, a presença de aminoácidos excitotóxicos como o glutamato, a elevada concentração de ferro em várias áreas do cérebro, a característica das membranas celulares (ricas em ácidos graxos de cadeia lateral poli-insaturada), a considerável quantidade de microglia, capaz de produzir $\mathrm{O}_{2}^{--}$e $\mathrm{H}_{2} \mathrm{O}_{2}$ quando ativada, e a escassez das defesas antioxidantes são alguns dos principais fatores relacionados com a suscetibilidade do cérebro ao dano oxidativo ${ }^{29}$.

Para manter a homeostasia, existem mecanismos de defesa conhecidos como antioxidantes, os quais podem ser enzimáticos ou não enzimáticos. Os componentes do sistema de defesa antioxidante enzimático atuam catalisando as oxidações de outras moléculas e incluem, entre outros, a enzima superóxido dismutase (SOD), que converte os radicais superóxido em peróxido de hidrogênio, a glutationa peroxidase (GPX) e a catalase (CAT), que decompõem o peróxido de hidrogênio. Os componentes antioxidantes não enzimáticos que atuam removendo as ER e prevenindo reações em cadeia incluem, por exemplo, o alfa-tocoferol (vitamina E), o betacaroteno, o ascorbato (vitamina C) e a glutationa ${ }^{30-32}$. Apesar de a manutenção das funções celulares depender de uma produção basal de ER, a produção destas em excesso pode comprometer a capacidade dos sistemas de defesa em neutralizar esses intermediários reativos, ocasionando uma condição denominada estresse oxidativo $^{33}$. No diabetes, o prejuízo na absorção de glicose, particularmente em células do músculo e do tecido adiposo, resulta em hiperglicemia. Evidências indicam que a hiperglicemia ocasiona aumento da produção mitocondrial de ERO, podendo levar ao desequilíbrio redox na ausência de uma resposta compensatória adequada do sistema de defesa antioxidante ${ }^{34}$. Considerando que as células neuronais não dependem da insulina para captarem glicose, a hiperglicemia persistente no diabetes provoca um aumento acentuado na captação de glicose neuronal, ocasionando um fenômeno conhecido como neurotoxicidade ${ }^{35}$. Por meio da ativação de vias de sinalização sensíveis ao estado redox, incluindo as vias intracelulares do fator de transcrição nuclear kappa beta (NF-K $\beta$ ), proteína quinase p38 ativada por mitógenos (p38 mitogen-activated protein kinase, MAPK), JNK/proteínas quinases estresse-ativadas, proteína quinase $C$ (PKC) e produtos finais de glicação avançada (advanced glycation end products, AGE)/receptores para AGE (receptors for AGE, RAGE), o estresse oxidativo pode ocasionar uma série de disfunções e danos celulares responsáveis pelas complicações do diabetes ${ }^{34}$.

Os danos celulares decorrentes do excesso de ER podem atingir diferentes substratos na célula, causando oxidação de proteínas, do DNA, do RNA, ou peroxidação lipídica. No cérebro, os produtos da oxidação de ácidos graxos poli-insaturados, principalmente os abundantes ácidos araquidônico e decosahexanóico, são representados pelo malondialdeído e o 4-hidroxinonenal ${ }^{36}$. Considerando 
que os lipídeos são os principais componentes das membranas neuronais, assim como das bainhas de mielina, que ajudam a realizar a sinalização neuronal, a peroxidação lipídica pode resultar em comprometimento significativo da função cerebral ${ }^{37}$. Por apresentar um sistema de defesa antioxidante escasso, o cérebro está propenso ao estresse oxidativo. Como muitas doenças neurodegenerativas estão envolvidas com o aumento do dano oxidativo, com a disfunção mitocondrial e com falhas no sistema de reparo e substituição de biomoléculas oxidadas essenciais ao funcionamento do organismo ${ }^{38}$, a encefalopatia diabética, uma complicação do diabetes, também pode estar relacionada com o aumento da produção de ER e com o comprometimento das defesas antioxidantes.

A disfunção mitocondrial tem sido relacionada com muitas doenças, incluindo diabetes, por ser uma fonte endógena de ERO, observação que torna a mitocôndria alvo de investigação quanto ao dano oxidativo ${ }^{39}$. Em ratos diabéticos, a hiperglicemia está associada com alteração do metabolismo mitocondrial, resultando em acréscimos na produção de $\mathrm{O} 2 \cdot-\mathrm{e}$ de $\mathrm{H}_{2} \mathrm{O}_{2}$, além de aumento acentuado na medida de substâncias reativas ao ácido tiobarbitúrico (TBARS) em homogenatos de córtex cerebral, indicando a presença de peroxidação lipídica em fase inicial do diabetes ${ }^{39}$.

Alterações no sistema nervoso autônomo, conhecidas por neuropatias, são complicações comuns em diabéticos e têm sido relacionadas com biomarcadores de estresse oxidativo ${ }^{40}$. Em roedores, a exposição durante 10 dias a STZ, um modelo experimental de diabetes tipo 1, ocasiona prejuízo na transmissão e na plasticidade sináptica em isolados de gânglios cervicais, associado com redução de fator neurotrófico derivado do cérebro (brain-derived neutrophic factor, BDNF) e alteração de biomarcadores de estresse oxidativo, incluindo aumento da atividade das enzimas glutationa peroxidase (GPx), glutationa redutase, CAT e dos níveis de TBARS, bem como diminuição da razão glutationa reduzida/glutationa oxidada (GSH/GSSG), sugerindo uma possível correlação entre as manifestações do diabetes no sistema nervoso autônomo e o estresse oxidativo ${ }^{40}$.

Um estudo realizado por Haiching et al. ${ }^{41}$ demonstrou que ratos com diabetes induzida por STZ apresentam alterações estruturais e funcionais no hipocampo, levando ao comprometimento da memória e do aprendizado, em associação com a expressão diminuída no hipocampo das enzimas de defesa antioxidante GPx, SOD e CAT, somado ao aumento de malondialdeído, um marcador de peroxidação lipídica. Com a progressão da doença em roedores, o diabetes resulta em alterações patológicas dos vasos sanguíneos cerebrais e tecido neural, sendo essas alterações marcadas pelo estresse oxidativo e depleção das defesas antioxidantes SOD, CAT e GPx nas células neuronais ${ }^{42}$.

Buscando compreender a relação entre diabetes e depressão, pesquisas com modelo animal dessas patologias vêm demonstrando o envolvimento do estresse oxidativo com as alterações no SNC e o comportamento do tipo depressivo ${ }^{23,43}$. Em humanos, Michel et al. ${ }^{44}$ encontraram evidências clínicas de que o estresse oxidativo pode estar envolvido com a depressão ao avaliar homogenatos da região frontal do córtex (estudo postmortem) de pacientes com depressão comparados a indivíduos controles. Nesse estudo foi constatado um aumento na atividade da enzima antioxidante cobre/zinco superóxido dismutase (Cu/Zn SOD), indicativo de uma resposta compensatória ao estresse oxidativo ${ }^{44}$. Estudos anteriores indicaram uma correlação entre o dano oxidativo e os níveis de defesa antioxidante em pacientes com depressão, sendo que as alterações foram acompanhadas por uma redução nos níveis de ácido ascórbico (antioxidante que impede a peroxidação lipídica) e revertidas após o tratamento com os antidepressivos fluoxetina e citalopram ${ }^{45}$.

Entre os principais antioxidantes endógenos, destaca-se a GSH, cuja redução tem sido apontada como um dos fatores relacionados à maior vulnerabilidade do cérebro ao dano oxidativo em pacientes com encefalopatia diabética, assim como em pacientes com depressão ${ }^{46}$. A diminuição dos níveis de GSH em pacientes com depressão sugere que o comprometimento das defesas antioxidantes tem um papel importante na fisiopatologia do transtorno de humor ${ }^{47}$.

\section{DEPRESSÃO RELACIONADA À ENCEFALOPATIA DIABÉTICA}

A depressão é um transtorno mental comum em todo o mundo, com uma estimativa global de mais de 350 milhões de pessoas afetadas. Esse transtorno, que atinge todas as idades, se caracterizada por tristeza, perda de interesse ou prazer, sentimentos de culpa ou baixa autoestima, distúrbios do sono ou do apetite, sensação de cansaço e falta de concentração. Ainda, a depressão muitas vezes é acompanhada por sintomas de ansiedade. Esses problemas podem se tornar crônicos ou recorrentes, resultando em deficiências substanciais na capacidade de um indivíduo para cuidar de suas responsabilidades cotidianas. No desfecho mais grave, a depressão pode levar ao suicídio. Quase 1 milhão de vidas são perdidas anualmente devido ao suicídio, o que se traduz em 3.000 mortes por suicídio todos os dias ${ }^{48}$. 
Assim como o diabetes, a depressão está entre as principais causas de incapacidade funcional e possui alta incidência na população, contribuindo significativamente para a carga global de doenças, afetando pessoas de todas as comunidades em todo o mundo ${ }^{9}$. Considerando que a prevalência desse transtorno em diabéticos é superior ao da população saudável ${ }^{10}$, e que as estimativas para prevalência global de diabetes continuam aumentando, atualmente é provável que a população com depressão também tenha aumentado. De acordo com o International Diabetes Federation (IDF) as estimativas mais recentes indicam que 387 milhões de pessoas têm diabetes, e o número de pessoas com a doença tende a aumentar para além de 592 milhões em menos de 25 anos $^{3}$. No entanto, como muitos casos não são diagnosticados, um grande número de pacientes diabéticos está progredindo em direção às complicações da doença ${ }^{3}$. Os dados também revelam existir uma tendência de aparecimento do diabetes em pessoas cada vez mais jovens. Além disso, com $80 \%$ do número total da população afetada vivendo em países de baixa e média renda, onde a epidemia está em elevado crescimento, os números mais recentes do IDF fornecem uma indicação preocupante sobre o futuro impacto do diabetes para o desenvolvimento global ${ }^{3}$.

Os dados acima expostos são alarmantes, pois o diabetes é uma doença que progride com uma série de complicações, incluindo danos ao SNC que podem prejudicar a função cognitiva ${ }^{5}$. Na década de 20 já se reconhecia que o diabetes pode causar disfunção cognitiva. Posteriormente, no ano de 1950, o termo "encefalopatia diabética" foi introduzido para descrever o comprometimento cognitivo em pacientes diabéticos como uma das complicações da doença ${ }^{49}$. Desde então, estudos em pacientes diabéticos e em modelo experimental de diabetes tentam elucidar o mecanismo relacionado à disfunção cognitiva e o elevado risco de depressão nesses pacientes. Nesse contexto, os trabalhos de Biessels et al. têm contribuído para o entendimento do impacto do diabetes no SNC, uma vez que o cérebro é suscetível aos efeitos dessa doença ${ }^{6,7}$. A encefalopatia diabética é caracterizada por prejuízo das funções cognitivas e deficits neurocomportamentais ${ }^{7}$.

As alterações decorrentes da encefalopatia diabética terão impacto no processo de aprendizado, memória, resolução de problemas, proposta informativa, mental e velocidade motora. Além disso, os distúrbios cognitivos específicos associados com o diabetes foram descritos recentemente ${ }^{50}$. Deficiências foram repetidamente observadas em domínios cognitivos particulares, incluindo a memória e a psicomotricidade. Há alguma sobreposição nas conclusões a respeito das alterações cognitivas no diabetes, mas um risco aumentado de demência ou comprometimento cognitivo leve está descrito ${ }^{51}$. Os pacientes diabéticos sofrem de sintomas significativamente mais introversivos do que pessoas saudáveis, especialmente sintomas somáticos, perturbação do sono, compulsões e humor depressivo ${ }^{51}$. As discussões mais recentes sugerem que alterações metabólicas do diabetes, somadas à condição inflamatória da doença, podem estar relacionadas aos sintomas somático-afetivos da depressão, como distúrbios do sono, mudança de apetite, fadiga e problemas psicomotores ${ }^{52}$.

Sabe-se que pessoas com diabetes correm um risco substancialmente aumentado de experimentar angústia mental, particularmente depressão ${ }^{53}$. Além disso, um crescente conjunto de evidências indicam uma relação bidirecional entre essas duas condições ${ }^{52,54}$. No entanto, a depressão muitas vezes é subdiagnosticada em pessoas com diabetes ${ }^{55}$. Entre o período de 2004-2006, uma pesquisa a respeito do diabetes tipo 2 realizada com indivíduos idosos, incluindo 458 participantes com diabetes tipo 2 e 546 sem diabetes, demonstrou que pacientes idosos com diabetes têm mais que o dobro de probabilidade de apresentarem transtornos depressivos em comparação a indivíduos sem diabetes ${ }^{19}$. Uma metanálise conduzida por Anderson et al. ${ }^{10}$ com 42 publicações de estudos - uma amostra de 21.351 indivíduos adultos - levou à conclusão de que a prevalência da depressão em adultos com qualquer tipo de diabetes é o dobro da observada em indivíduos sem diabetes ${ }^{56}$.

As consequências emocionais do diabetes foram avaliadas em um estudo transversal em uma amostra randomizada de 264 indivíduos obtidos de 960 pacientes com diabetes tipo 2. O estudo demonstrou que a depressão é um problema de saúde comum em pacientes diabéticos tipo 2 , com uma prevalência de $13 \%{ }^{57}$. Na Índia, região com o segundo maior número de pessoas com diabetes tipo 2 no mundo, de acordo com o IDF 2014, um estudo prospectivo de caso-controle determinou uma prevalência de depressão em pacientes com diabetes superior à observada em pessoas sem a doença. Nesse mesmo estudo, também foi verificado que a ocorrência de depressão não está associada com controle glicêmico inadequado, duração do diabetes, ou mesmo com as complicações decorrentes da doença ${ }^{58}$. Em contraste, outros estudos com pacientes com diabetes tipo 2 associam a depressão ao baixo controle glicêmico e a complicações resultantes da progressão da doença ${ }^{19,59}$.

O diabetes e a depressão são condições debilitantes crônicas que estão associadas com significativa morbidade, mortalidade e custos em saúde. Ambas 
as doenças estão associadas de forma independente com um maior risco de demência e, quando existe a combinação das exposições, esse risco é muito elevado ${ }^{60}$. Outras consequências da ocorrência concomitante dessas doenças é a diminuição da adesão ao tratamento recomendado ${ }^{61}$, inadequado controle metabólico ${ }^{59}$, maiores complicações, diminuição da qualidade de vida, aumento da utilização de cuidados com a saúde e de custos, incapacidade e perda de rendimento, e aumento do risco de morte ${ }^{62}$. Contudo, a depressão é um fator de risco modificável e, sendo assim, uma intervenção adequada é especialmente importante em pacientes com complicações do diabetes ${ }^{19}$.

Holt et al. estudaram o quanto a carga psicológica do diabetes contribui para a depressão e constataram que a observação não foi suficiente para explicar a relação entre essas duas condições ${ }^{56}$. Pesquisas futuras sugerem uma nova abordagem de estudo que considere a influência de vias biológicas e comportamentais compartilhadas na predisposição a desordens metabólicas e afetivas ${ }^{56}$. A Figura 1 resume os mecanismos envolvidos na relação entre diabetes e depressão, apontando a encefalopatia diabética - uma importante consequência das complicações diabéticas - relacionada com a depressão.

\section{DANO OXIDATIVO RELACIONADO AO DIABETES E DEPRESSÃO}

Modelos animais experimentais de diabetes e depressão, como o modelo de diabetes induzida por STZ e o teste de natação forçada (forced swimming test, FST) em roedores, respectivamente, têm sido utilizados para compreender as alterações neurais e comportamentais envolvidas na encefalopatia diabética e os mecanismos de depressão e declínio cognitivo relacionado a essas patologias. O teste STZ é amplamente aceito como um modelo animal para o estudo da depressão, pois a imobilidade dos animais durante o teste é um fenômeno relativamente específico e capaz de reproduzir alguns aspectos da depressão em seres humanos ${ }^{63}$. Pesquisas anteriores demonstraram uma mudança comportamental semelhante à depressão, pois a duração do tempo de imobilidade no teste é maior em animais diabéticos quando comparados com animais controles s $^{23,43,63-66}$. No mesmo modelo experimental, também tem sido relatado o envolvimento do estresse oxidativo nas complicações decorrentes do diabetes ${ }^{23,43}$. Evidências, tanto em estudos experimentais como em estudos clínicos, sugerem que o estresse oxidativo ${ }^{67}$ e as

\section{VIAS BIOLÓGICAS COMUNS INTER-RELACIONADAS}

\section{Alterações Disfunção do Eixo Inflamação Distúrbio Epigenéticas Hipotálamo-Hipófise-Adrenal Estresse Oxidativo Sono/Ritmo Circadiano}

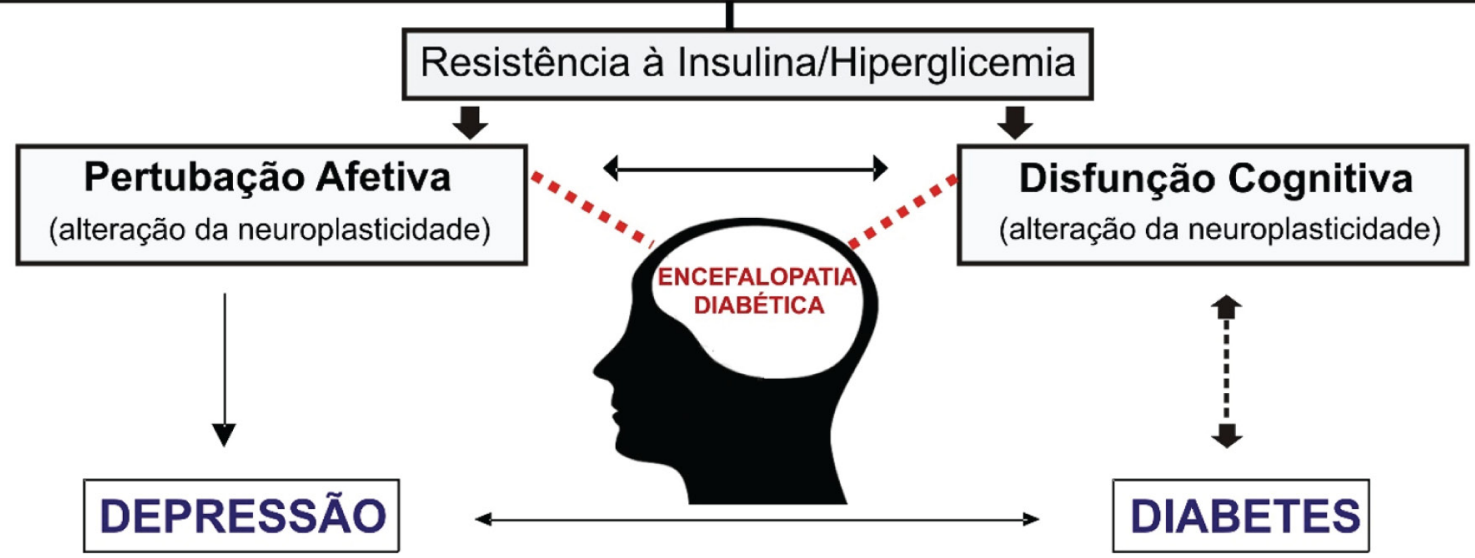

Figura 1: Vias biológicas inter-relacionadas no diabetes e depressão: mudanças hereditárias na expressão gênica, sob a influência do ambiente (alterações epigenéticas); disfunção do eixo hipotálamo-hipófise-adrenal que se manifesta por alterações do cortisol; modificações do padrão do sono associadas com depressão e alterações do ritmo circadiano como risco para diabetes tipo 2; inflamação crônica evidenciada em pacientes diabéticos e associada à depressão; alterações na ação da insulina relacionadas com modificações na neuroplasticidade identificadas tanto no diabetes como na depressão. As linhas em vermelho indicam a coexistência dessas doenças, uma condição denominada encefalopatia diabética. As setas de ponta dupla indicam vias bidirecionais (modificado de Holt et al. ${ }^{56}$ ). 
EROS desempenham um papel importante no desenvolvimento das complicações diabéticas ${ }^{68}$.

Uma pesquisa conduzida por Wayhs et al. ${ }^{23}$ verificou a ocorrência de dano a lipídeos e proteínas em modelo animal de diabetes/depressão, mostrando que a combinação terapêutica de insulina e clonazepam protege contra o dano oxidativo nesse modelo experimental. O clonazepam é utilizado para tratar a depressão em pacientes diabéticos, já tendo sido demonstrado em modelo animal que esse medicamento reverte o tempo de imobilidade no FST, tem efeito antidepressivo e apresenta um efeito inibitório sobre a produção de $\mathrm{ER}^{43}$. Ainda, a combinação de insulina e clonazepam demonstrou ser efetiva em reverter o prolongado tempo de imobilidade nos animais diabéticos, levando-os a retornar ao padrão de comportamento dos animais sem diabetes ${ }^{23}$. Anteriormente, Rajtar et al. ${ }^{69}$ verificaram que o clonazepam exerce um efeito inibitório in vitro sobre a produção de ERO, sugerindo que o clonazepam possa causar uma diminuição da ativação plaquetária e da liberação de mediadores pró-inflamatórios por neutrófilos ativados.

A insulina tem efeito na função cognitiva ${ }^{70}$, devido à sua ação neuroprotetora em condições diabéticas e oxidativas ${ }^{71}$. A produção excessiva de radicais livres de oxigênio que pode ocorrer em pacientes com diabetes induz a uma variedade de lesões em macromoléculas, incluindo o DNA. Uma pesquisa utilizando o ensaio de cometa para investigar danos no DNA em leucócitos de ratos diabéticos submetidos ao FST mostrou que o tratamento agudo com insulina reduz o índice de dano ao DNA quando comparado aos controles, tendo sido observada uma correlação positiva entre a glicemia e o índice de dano ao DNA ${ }^{72}$. Considerando que a hiperglicemia é um estado patológico crônico que leva ao estresse oxidativo, o efeito da insulina em reduzir o dano ao DNA pode ser devido à melhora da glicemia ${ }^{73}$. Em humanos, a insulina e o receptor de insulina têm sido associados com a função cognitiva e o envelhecimento ${ }^{74}$. Estudos anteriores com o mesmo modelo animal experimental mostraram que a insulina impede o dano neuronal no córtex ${ }^{75}$. O dano ao DNA já foi verificado no córtex pré-frontal, hipocampo e estriado em modelo experimental de diabetes/depressão, e a correlação positiva entre a glicemia e o índice de peroxidação lipídica foi observada no córtex pré-frontal e hipocampo dos animais, sugerindo que esse é um processo de oxirredução alterado ${ }^{66}$. Em roedores, o tratamento com insulina e/ou clonazepam foi capaz de proteger contra esses danos ${ }^{66}$.

A destruição de neurônios devido ao estresse oxidativo pode ser uma das causas de depressão no diabetes. No córtex pré-frontal de animais diabéticos por STZ submetidos ao FST foi demonstrado um aumento significativo de TBARS, um parâmetro de peroxidação lipídica, e foi verificada no plasma desses animais uma diminuição significativa da reatividade antioxidante total, indicando que o estresse oxidativo pode estar envolvido nos sintomas da encefalopatia diabética ${ }^{43}$. Uma pesquisa realizada por Kamboj \& Sandhir ${ }^{76}$ encontrou uma associação entre o aumento do estresse oxidativo mitocondrial e os níveis de caspase-3 ativa. Ainda, análises de microscopia eletrônica revelaram mudanças na morfologia da mitocôndria e condensação da cromatina em neurônios de animais diabéticos, sendo esse um parâmetro de avaliação de morte celular.

Como a mitocôndria é uma fonte de EROS, a disfunção mitocondrial pode estar relacionada com as alterações observadas na encefalopatia diabética. $O$ estresse oxidativo parece ter um papel no dano cerebral no diabetes, uma vez que a hiperglicemia crônica acentua o estresse oxidativo ao provocar um aumento da produção de EROS e da peroxidação lipídica e, além disso, reduzir a ação das enzimas mitocondriais NADH desidrogenase, succinato desidrogenase e citocromo oxidase, levando a uma deficiência da função mitocondrial ${ }^{76}$.

\section{ANTIOXIDANTES E ENCEFALOPATIA DIABÉTICA}

Novas estratégias para a prevenção e tratamento das complicações do diabetes são constantemente investigadas. A insulina e os hipoglicemiantes, que são tradicionalmente utilizados no tratamento do diabetes e suas complicações, apresentam entre seus efeitos uma ação antioxidante, como já mostrado em diversos estudos ${ }^{23,71,72,77}$. Sendo assim, as pesquisas atuais com modelo experimental de diabetes têm buscado novos antioxidantes como uma abordagem terapêutica para o tratamento ou prevenção da encefalopatia diabética.

O diabetes induz dano a neurônios, aumento de células apoptóticas no hipocampo, e disfunção cognitiva acompanhados de aumento acentuado do estresse oxidativo no cérebro ${ }^{78}$. O catalpol, por apresentar propriedades anti-inflamatórias e antioxidantes, foi testado por meio da suplementação oral em longo prazo em ratos diabéticos e mostrou capacidade para proteger os neurônios contra a neurodegeneração ao promover melhora da atividade de enzimas antioxidantes GPx, SOD, CAT e diminuir a concentração de malondialdeído (MDA ${ }^{78}$. Em modelo animal experimental de diabetes os níveis de MDA estão aumentados. Esse produto tóxico resulta dos efeitos danosos que o excesso de radicais livres exerce sobre as membranas celulares. Além 
disso, neste modelo animal a GPx e a GSH estão reduzidas e há um aumento do número de células positivas para o 4-hidroxinonenal, indicador de lipoperoxidação, ocasionando dano aos neurônios do córtex ${ }^{77}$. O ácido docosahexaenóico (DHA) é um ácido graxo poli-insaturado de cadeia longa (ômega 3) cuja ação neuroprotetora mostrou ser eficaz em atenuar as alterações oxidativas no córtex pré-frontal de ratos diabéticos, sendo seu efeito comparável ao da insulina no mesmo estudo ${ }^{77}$.

Asuscetibilidade dos neurônios e da microvasculatura cerebral ao estresse oxidativo está relacionada às alterações que contribuem para encefalopatia diabética primária ${ }^{79}$. Nesse contexto, um estudo investigando o papel do estresse oxidativo no endotélio microvascular cerebral humano, sua relação com o status de GSH celular e a sinalização apoptótica mitocondrial mostrou que tanto sensibilizadores da insulina quanto agentes antioxidantes tióis ( $\mathrm{N}$-acetilcisteína) fornecem proteção celular ao restaurar o equilíbrio do status de GSH, preservando a integridade endotelial ${ }^{79}$.

Outro composto que demonstra potencial terapêutico na encefalopatia diabética é o paeonol, componente ativo isolado da casca da raiz da Paeonia suffruticosa, planta utilizada pela medicina chinesa que demonstra potencial capacidade neuroprotetora e hipoglicemiante ${ }^{80}$. O paeonol mostrou atenuar o estresse oxidativo na encefalopatia diabética em ratos STZ, impedindo o prejuízo cognitivo, promovendo a diminuição na expressão do RAGE e do NF-KB no hipocampo e córtex, reduzindo o acúmulo de AGEs, o que pode estar relacionado com a inibição da via AGEs/RAGE/NF-KB envolvida com as complicações crônicas do diabetes ${ }^{80}$. Como já discutido por Lu et al. ${ }^{81}$, a via AGEs/RAGE/NF-KB, crítica para o estresse oxidativo, está envolvida com a patogênese da encefalopatia diabética. Logo, os antioxidantes podem trazer benefícios para a saúde do cérebro, e sugere-se como potencial alvo terapêutico as proantocianidinas extraídas da semente da uva, cujas propriedades parecem modular vias redox sensíveis.

Acurcumina, um componente ativo do açafrão-da-índia, possui propriedades anti-inflamatórias e antioxidantes bem estabelecidas, mostrando uma ação neuroprotetora e um potencial benefício para o tratamento e prevenção da encefalopatia diabética, visto que atenua o estresse oxidativo e a disfunção cognitiva em ratos diabéticos ${ }^{82}$. Um estudo demostrou que o extrato é eficaz contra o estresse oxidativo, aumenta o teor de GSH e a atividade da SOD e CAT no hipocampo, reduz a hiperglicemia, diminui os níveis de AGE e proteínas glicadas e reduz a produção de citocinas pró-inflamatórias, promovendo melhora da cognição nos animais ${ }^{83}$

A produção excessiva de ER, característica do estresse oxidativo, é um fator importante que induz a morte de neurônios ${ }^{84}$. A hiperglicemia, além do estresse oxidativo mitocondrial, causa disfunções que podem contribuir para o dano neuronal evidenciado na encefalopatia diabética. Foi observado que a suplementação com $\mathrm{N}$-acetilcisteína, um composto tiólico com ação antioxidante, consegue reverter alterações decorrentes da hiperglicemia, restaura o funcionamento de mitocôndrias, o que reduz o estresse oxidativo mitocondrial, atua na ativação de vias apoptóticas relacionadas com a morte de células neuronais impedindo a liberação do citocromo $\mathrm{C}$ e a ativação da caspase-3, sugerindo um potencial benefício como terapia adjuvante aos anti-hiperglicemiantes tradicionais ${ }^{76}$. Com estrutura molecular semelhante ao catalpol e de forma similar com eficaz ação antiapoptótica, a acubina possui função neuroprotetora associada ao favorecimento da atividade de enzimas antioxidantes endógenas e a capacidade de eliminar o excesso de ER e proteger contra o dano a lipídeos de membrana, melhorando a glicemia em modelo experimental de encefalopatia diabética ${ }^{84}$. Dados de estudos experimentais anteriores demonstram que a acubina pode evitar a apoptose modulando a expressão de genes relacionados a apoptose $\mathrm{Bcl}-2$ e $\mathrm{Bax}^{85}$. O efeito neuroprotetor, a melhora da cognição e proteção contra a perda de neurônios ocorre tanto em curto quanto em longo prazo no tratamento experimental com acubina ${ }^{86}$.

\section{CONCLUSÃO}

A associação entre diabetes e depressão é um grave problema de saúde pública. Ambas as doenças apresentam elevada ocorrência na população mundial, e pesquisas indicam que essas patologias estão associadas. Atualmente, considera-se que vias biológicas comuns, incluindo via redox sensível, são responsáveis por essa associação. Dados de estudos dos últimos 10 anos sugerem que o estresse oxidativo, por ocasionar danos ao SNC, pode exercer um papel fundamental na relação entre encefalopatia diabética e depressão. Nesse sentido, é necessária uma melhor compreensão a respeito dessa relação, pois poucos são os estudos em humanos que abordam a depressão relacionada à encefalopatia diabética. As pesquisas em modelo experimental têm fornecido um conhecimento relevante sobre o mecanismo biológico envolvido e indicam, como perspectiva, a necessidade de estudos que abordem novas possibilidades de alvos terapêuticos para a 
prevenção e tratamento da encefalopatia diabética, incluindo abordagens com antioxidantes, mas é preciso investigar seus benefícios em humanos. Futuros estudos investigando a associação de insulina ou hipoglicemiantes orais com moléculas que promovam prevenção de processos oxidativos em pacientes portadores de diabetes tipo 1 e 2 serão promissores.

\section{Conflito de interesse}

Os autores declaram não haver conflito de interesse.

\section{REFERÊNCIAS}

1. Kerner W, Brückel J, German Diabetes Association. Definition, classification and diagnosis of diabetes mellitus. Exp Clin Endocrinol Diabetes. 2014;122(7):384-6. http:// dx.doi.org/10.1055/s-0034-1366278 PMid:25014088.

2. American Diabetes Association (ADA) Standards of medical care in diabetes - 2013. Diabetes Care. 2013;36(Suppl 1):S11-66. http://dx.doi.org/10.2337/ dc13-S011. PMid:23264422.

3. International Diabetes Federation. Diabetes Atlas 2013. 6. ed. Brussels; 2013 [citado em 2015 Ago 22]. Disponível em: http://www.idf.org/ sites/default/files/Atlas-poster-2014_ EN.pdf.

4. American Diabetes Association (ADA). Classification and diagnosis of diabetes. Diabetes Care. 2015;38(Suppl 1):S8-16. http:// dx.doi.org/10.2337/dc15-S005. PMid:25537714.

5. Artola A. Diabetes-, stress- and ageing-related changes in synaptic plasticity in hippocampus and neocortex--the same metaplastic process? Eur J Pharmacol. 2008;585(1):153-62. http://dx.doi. org/10.1016/j.ejphar.2007.11.084. PMid:18395200.

6. Biessels GJ, Gispen HW. The impact of diabetes on cognition: What can be learned from rodent models? Neurobiol Aging. 2005;26(Suppl 1):36-41. http://dx.doi.org/10.1016/j. neurobiolaging.2005.08.015. PMid:16223548.

7. Biessels GJ, van der Heide LP, Kamal A, Bleys RL, Gispen WH. Ageing and diabetes: implications for brain function. Eur J Pharmacol. 2002;441(1-2):1-14. http://dx.doi. org/10.1016/S0014-2999(02)01486-3. PMid:12007915.

8. Jongen C, Biessels GJ. Structural brain imaging in diabetes: a methodological perspective. Eur
J Pharmacol. 2008;585(1):208-

18. http://dx.doi.org/10.1016/j. ejphar.2007.11.085. PMid:18407264.

9. Egede LE. Effect of depression on self-management behaviors and health outcomes in adults with type 2 diabetes. Curr Diabetes Rev. 2005;1(3):235-43. http://dx.doi. org/10.2174/157339905774574356. PMid:18220600.

10. Anderson RJ, Freedland KE, Clouse RE, Lustman PJ. The prevalence of comorbid depression in adults with diabetes: a meta-analysis. Diabetes Care. 2001;24(6):1069-78. http:// dx.doi.org/10.2337/diacare.24.6.1069. PMid:11375373.

11. Egede LE. Diabetes,major depression, and functional disability among U.S. adults. Diabetes Care. 2004;27(2):421-8. http://dx.doi. org/10.2337/diacare.27.2.421. PMid:14747223.

12. Riley AA, McEntee LM, Gerson L, Dennison RC. Depression as a comorbidity to diabetes: implications for management. $J$ Nurse Pract. 2009;5(7):523-35. http://dx.doi. org/10.1016/j.nurpra.2009.03.020.

13. Maes M, Kubera M, Obuchowiczwa E, Goehler L, Brzeszcz J. Depression's multiple comorbidities explained by (neuro)inflammatory and oxidative \& nitrosative stress pathways. Neuroendocrinol Lett. 2011;32(1):724. PMid:21407167.

14. Kumar P, Rao GN, Pal BB, Pal A. Hyperglycemia-induced oxidative stress induces apoptosis by inhibiting PI3-kinase/Akt and ERK1/2 MAPK mediated signaling pathway causing downregulation of 8-oxoG-DNA glycosylase levels in glial cells. Int J Biochem Cell Biol. 2014;53:30219. http://dx.doi.org/10.1016/j. biocel.2014.05.038. PMid:24907397.

15. Ola MS, Aleisa AM, Al-Rejaie SS, Abuohashish HM, Parmar MY, Alhomida AS, et al. Flavonoid, morin inhibits oxidative stress, inflammation and enhances neurotrophic support in the brain of streptozotocininduced diabetic rats. Neurol Sci. 2014;35(7):1003-8. http://dx.doi. org/10.1007/s10072-014-1628-5. PMid:24413816.

16. Valko M, Leibfritz D, Moncol J, Cronin MT, Mazur M, Telser J. Free radicals and antioxidants in normal physiological functions and human disease. Int J Biochem Cell Biol. 2007;39(1):44-84. http://dx.doi. org/10.1016/j.biocel.2006.07.001. PMid:16978905.

17. Mastrocola R, Restivo F, Vercellinatto I, Danni O, Brignardello E, Aragno $\mathrm{M}$, et al. Oxidative and nitrosative stress in brain mitochondria of diabetic rats. J Endocrinol. 2005;187(1):37-44. http://dx.doi.org/10.1677/joe.1.06269. PMid:16214939.

18. Liu J, Wang S, Feng L, Ma D, Fu $Q$, Song $Y$, et al. Hypoglycemic and antioxidant activities of paeonol and its beneficial effect on diabetic encephalopathy in streptozotocininduced diabetic rats. $J$ Med Food. 2013;16(7):577-86. http:// dx.doi.org/10.1089/jmf.2012.2654. PMid:23875897.

19. Shehatah A, Rabie MA, Al-Shahry A. Prevalence and correlates of depressive disorders in elderly with type 2 diabetes in primary health care settings. J Affect Disord. 2010;123(13):197-201. http://dx.doi.org/10.1016/j. jad.2009.09.002. PMid:19804911.

20. Liu Z, Zhang J, Yan J, Wang Y, Li Y. Leucocyte telomere shortening in relation to newly diagnosed type 2 diabetic patients with depression. Oxid Med Cell Longev. 2014;(2014):1-8. http://dx.doi.org/10.1155/2014/673959.

21. Gawryluk JW, Wang JF, Andreazza AC, Shao L, Young LT. Decreased levels of glutathione, the major brain antioxidant, in post-mortem prefrontal cortex from patients with psychiatric disorders. Int 
$J$ Neuropsychopharmacol. 2011;14(1):123-30. http://dx.doi. org/10.1017/S1461145710000805. PMid:20633320.

22. Najjar S, Pearlman DM, Devinsky O, Najjar A, Zagzag D. Neurovascular unit dysfunction with blood-brain barrier hyperpermeability contributes to major depressive disorder: a review of clinical and experimental evidence. J Neuroinflammation. 2013;10:142. http://dx.doi.org/10.1186/1742-209410-142. PMid:24289502.

23. Wayhs CA, Manfredini V, Sitta A, Deon M, Ribas G, Vanzin C, et al. Protein and lipid oxidative damage in streptozotocin-induced diabetic rats submitted to forced swimming test: the insulin and clonazepam effect. Metab Brain Dis. 2010;25(3):297-304. http:// dx.doi.org/10.1007/s11011-010-92110. PMid:20838862.

24. Bouayed J, Rammal H, Soulimani R. Oxidative stress and anxiety: relationship and cellular pathways. Oxid Med Cell Longev. 2009;2(2):637. http://dx.doi.org/10.4161/ oxim.2.2.7944. PMid:20357926.

25. Smith DG, Cappai R, Barnham KJ. The redox chemistry of the Alzheimer's disease amyloid $\beta$ peptide. Biochim Biophys Acta. 2007;1768(8):1976-90. http://dx.doi. org/10.1016/j.bbamem.2007.02.002. PMid:17433250.

26. Wang JF, Shao L, Sun X, Young LT. Increased oxidative stress in the anterior cingulate cortex of subjects with bipolar disorder and schizophrenia. Bipolar Disord. 2009;11(5):523-9. http:// dx.doi.org/10.1111/j.13995618.2009.00717.x. PMid:19624391.

27. Salim S. Oxidative stress and psychological disorders. Curr Neuropharmacol. 2014;12(2):1407. http://dx.doi.org/10.2174/15 70159X11666131120230309. PMid:24669208.

28. Adibhatla RM, Hatcher JF. Lipid oxidation and peroxidation in CNS health and disease: from molecular mechanisms to therapeutic opportunities. Antioxid Redox Signal. 2010;12(1):125-69. http:// dx.doi.org/10.1089/ars.2009.2668. PMid:19624272.

29. Halliwell B. Reactive species and antioxidants. Redox biology is a fundamental theme of aerobic life. Plant Physiol. 2006;141(2):312-
22. http://dx.doi.org/10.1104/ pp.106.077073. PMid:16760481.

30. Dröge W. Free radicals in the physiological control of cell function. Physiol Rev. 2002;82(1):4795. http://dx.doi.org/10.1152/ physrev.00018.2001. PMid:11773609.

31. Halliwell B. The wanderings of free radical. Free Radic Biol Med. 2009;46(5):53142. http://dx.doi.org/10.1016/j. freeradbiomed.2008.11.008. PMid:19111608.

32. Gutteridge JM, Halliwel B. Antioxidants: molecules, medicines, and myths. Biochem Biophys Res Commun. 2010;393(4):561-4. http:// dx.doi.org/10.1016/j.bbrc.2010.02.071. PMid:20171167.

33. Stone JR, Yang S. Hydrogen peroxide: a signaling messenger. Antioxid Redox Signal. 2006;8(34):243-70. http://dx.doi.org/10.1089/ ars.2006.8.243. PMid:16677071.

34. Evans JL, Goldfine ID, Maddux BA, Grodsky GM. Oxidative stress and stress-activated signaling pathways: a unifying hypothesis of type 2 diabetes. Endocr Rev. 2002;23(5):599-622. http://dx.doi.org/10.1210/er.20010039. PMid: 12372842.

35. Tomlinson DR, Gardiner NJ. Glucose neurotoxicity. Nat Rev Neurosci. 2008;9(1):36-45. http://dx.doi. org/10.1038/nrn2294. PMid:18094705.

36. Gandhi S, Abramov AY. Mechanism of oxidative stress in neurodegeneration. Oxid Med Cell Longev. 2012;2012:428010. http:// dx.doi.org/10.1155/2012/428010. PMid:22685618.

37. Joshi YB, Praticò D. Lipid peroxidation in psychiatric illness: overview of clinical evidence. Oxid Med Cell Longev. 2014;2014:828702.

38. Halliwell B. Oxidative stress and neurodegeneration: where are we now? J Neurochem. 2006;97(6):163458. http://dx.doi.org/10.1111/j.14714159.2006.03907.x. PMid:16805774.

39. Ortiz MC, Lores-Arnaiz S, Albertoni Borghese MF, Balonga S, Lavagna A, Filipuzzi AL, et al. Mitochondrial dysfunction in brain cortex mitochondria of STZ-diabetic rats: effect of I-Arginine. Neurochem Res. 2013;38(12):2570-80. http://dx.doi. org/10.1007/s11064-013-1172-3. PMid:24190597.
40. Alzoubi KH, Khabour OF, Alhaidar IA, Aleisa AM, Alkadhi KA. Diabetes impairs synaptic plasticity in the superior cervical ganglion: possible role for BDNF and oxidative stress. J Mol Neurosci. 2013;51(3):763-70. http://dx.doi.org/10.1007/s12031-0130061-1. PMid:23832486.

41. Zhou H, Liu J, Ren L, Liu W, Xing Q, Men $L$, et al. Relationship between spatial memory in diabetic rats and protein kinase $\mathrm{CY}$, caveolin-1 in the hippocampus and neuroprotective effect of catalpol. Chin Med J (Engl). 2014;127(5):916-23. PMid:24571888.

42. Yang $H$, Fan S, Song D, Wang Z, Ma S, Li S, et al. Long-term streptozotocin-induced diabetes in rats leads to severe damage of brain blood vessels and neurons via enhanced oxidative stress. Mol Med Rep. 2013;7(2):431-40. PMid:23232924.

43. Haeser AS, Sitta A, Barschak AG, Deon M, Barden AT, Schmitt $\mathrm{GO}$, et al. Oxidative stress parameters in diabetic rats submitted to forced swimming test: the clonazepam effect. Brain Res. 2007;1154:137-43. http://dx.doi. org/10.1016/j.brainres.2007.03.088. PMid:17490624.

44. Michel TM, Frangou S, Thiemeyer D, Camara S, Jecel J, Nara K, et al. Evidence for oxidative stress in the frontal cortex in patients with recurrent depressive disorder--a postmortem study. Psychiatry Res. 2007;151(1-2):145-50. http://dx.doi. org/10.1016/j.psychres.2006.04.013. PMid:17296234.

45. Khanzode SD, Dakhale GN, Khanzode SS, Saoji A, Palasodkar R. Oxidative damage and major depression: the potential antioxidant action of selective serotonin reuptake inhibitors. Redox Rep. 2003;8(6):365-70. http://dx.doi. org/10.1179/135100003225003393. PMid:14980069.

46. Gawryluk JW, Wang JF, Andreazza AC, Shao L, Yatham LN, Young LT. Prefrontal cortex glutathione S-transferase levels in patients with bipolar disorder, major depression and schizophrenia. Int J Neuropsychopharmacol. 2011;14(8):1069-74. http://dx.doi. org/10.1017/S1461145711000617. PMid:21733244.

47. Godlewska BR, Near J, Cowen PJ. Neurochemistry of major depression: a study using magnetic resonance 
spectroscopy. Psychopharmacology (Berl). 2015;232(3):501-7. http:// dx.doi.org/10.1007/s00213-0143687-y. PMid:25074444.

48. World Health Organization Depression (WHO). Fact sheet $\mathrm{N}^{\circ}$ 369. WHO; 2015 [citado em 2015 Ago 17]. Disponível em: http://www.who.int/ mediacentre/factsheets/fs369/en/.

49. Mijnhout GS, Scheltens P, Diamant M, Biessels GJ, Wessels AM, Simsek $S$, et al. Diabetic encephalopathy: A concept in need of a definition. Diabetologia. 2006;49(6):1447-8. http://dx.doi.org/10.1007/s00125-0060221-8. PMid:16598451.

50. Ma F, Wang T, Yin J, Bai XJ, Zhang $X D$, Meng J, et al. A case-control study on the influencing factors to mild cognitive impairment among the community-based elderly population. Liu Zhonghua Xing Bing Xue Za Zhi. 2008;29(9):873-7. PMID:19173847.

51. Cai XJ, Xu HQ, Lu Y. C-peptide and diabetic encephalopathy. Chin Med Sci J. 2011;26(2):119-25. http://dx.doi. org/10.1016/S1001-9294(11)60031-X. PMid:21703121.

52. Wiltink J, Michal M, Wild PS, Schneider A, König J, Blettner M, et al Associations between depression and diabetes in the community: do symptom dimensions matter? Results from the Gutenberg Health Study. PLoS One. 2014;9(8):e105499. http://dx.doi.org/10.1371/journal. pone.0105499. PMid:25127227.

53. Bouwman V, Adriaanse MC, van 't Riet E, Snoek FJ, Dekker JM, Nijpels G. Depression, anxiety and glucose metabolism in the general dutch population: the new Hoorn study. PLoS One. 2010;5(4):e9971. http://dx.doi.org/10.1371/journal. pone.0009971. PMid:20376307.

54. Golden SH, Lazo M, Carnethon M, Bertoni AG, Schreiner PJ, Diez Roux $\mathrm{AV}$, et al. Examining a bidirectional association between depressive symptoms and diabetes. JAMA. 2008;299(23):2751-9. http://dx.doi. org/10.1001/jama.299.23.2751. PMid:18560002.

55. Lloyd C. The effects of diabetes on depression and depression on diabetes. Diabetes Voice. 2008;53(10):23-6.

56. Holt RI, de Groot M, Lucki I, Hunter CM, Sartorius N, Golden SH. NIDDK International Conference Report on
Diabetes and Depression: Current Understanding and Future Directions. Diabetes Care. 2014;37(8):2067-77. http://dx.doi.org/10.2337/dc13-2134. PMid:25061135.

57. Dejenie Habtewold T, Radie YT, Sharew NT. Prevalence of depression among type 2 diabetic outpatients in black lion general specialized hospital, Addis Ababa, Ethiopia. Depres Res Treat. 2015;2015:1-8. http://dx.doi. org/10.1155/2015/184902.

58. Siddiqui S, Jha S, Waghdhare S, Agarwal NB, Singh K. Prevalence of depression in patients with type 2 diabetes attending an outpatient clinic in India. Postgrad Med J. 2014;90(1068):5526. http://dx.doi.org/10.1136/ postgradmedj-2014-132593. PMid:25092455.

59. Lustman PJ, Anderson RJ, Freedland KE, de Groot M, Carney RM, Clouse $\mathrm{RE}$. Depression and poor glycemic control: a meta-analytic review of the literature. Diabetes Care. 2000;23(7):934-42. http://dx.doi. org/10.2337/diacare.23.7.934. PMid:10895843.

60. Katon W, Pedersen HS, Ribe AR, Fenger-Grøn M, Davydow $D$, Waldorff FB, et al. Effect of depression and diabetes mellitus on the risk for dementia: a national population-based cohort study. JAMA Psychiatry. 2015;72(6):6129. http://dx.doi.org/10.1001/ jamapsychiatry.2015.0082. PMid:25875310.

61. Gonzalez JS, Peyrot M, McCarl LA, Collins EM, Serpa L, Mimiaga $M J$, et al. Depression and diabetes treatment nonadherence: $\mathrm{A}$ meta-Analysis. Diabetes Care. 2008;31(12):2398-403. http:// dx.doi.org/10.2337/dc08-1341. PMid:19033420.

62. Egede LE, Ellis C. Diabetes and depression: global perspectives. Diabetes Res Clin Pract. 2010;87(3):302-12. http://dx.doi. org/10.1016/j.diabres.2010.01.024. PMid:20181405.

63. Gomez R, Barros HM. Ethopharmacology of the antidepressant effect of clonazepam in diabetic rats. Pharmacol Biochem Behav. 2000;66(2):329-35. http:// dx.doi.org/10.1016/S00913057(00)00221-5. PMid:10880686.
64. Gomez R, Asnis N, Tannhauser SL, Barros HM. GABA agonists differentially modify blood glucose levels of diabetic rats. Jpn J Pharmacol. 1999;80(4):327-31. http://dx.doi.org/10.1254/jjp.80.327. PMid:10496333.

65. Caletti G, Olguins DB, Pedrollo EF, Barros HM, Gomez R. Antidepressant effect of taurine in diabetic rats. Amino Acids. 2012;43(4):1525-33. http:// dx.doi.org/10.1007/s00726-0121226-x. PMid:22302366.

66. Wayhs CA, Mescka CP, Vanzin CS, Ribas GS, Guerreiro G, Nin $\mathrm{MS}$, et al. Brain effect of insulin and clonazepam in diabetic rats under depressive-like behavior. Metab Brain Dis. 2013;28(4):563-70. http://dx.doi. org/10.1007/s11011-013-9397-z. PMid:23532706.

67. Maritim AC, Sanders RA, Watkins JB 3RD. Diabetes, oxidative stress, and antioxidants: a review. J Biochem Mol Toxicol. 2003;17(1):24-38. http://dx.doi.org/10.1002/jbt.10058. PMid:12616644.

68. Imaeda V, Aoki T, Kondo Y, Hori M, Ogata M, Obayashi H, et al. Protective effects of fluvastatin against reactive oxygen species induced DNA damage and mutagenesis. Free Radic Res. 2001;34(1):33-44. http://dx.doi. org/10.1080/10715760100300041. PMid:11234994.

69. Rajtar G, Zolkowska D, Keinrok Z. Effect of diazepam and clonazepam on the function of isolated rat platelet and neutrophils. Intern Med J Exp Clin Res. 2002;8(4):37-44.

70. Laron Z. Insulin and the brain. Arch Physiol Biochem. 2009;115(2):112-6. http://dx.doi. org/10.1080/13813450902949012. PMid:19485707.

71. Duarte AI, Santos MS, Seica R, Oliveira CR. Oxidative stress affects synaptosomal gammaaminobutyric acid and glutamate transport in diabetic rats: the role of insulin. Diabetes. 2004;53(8):21106. http://dx.doi.org/10.2337/ diabetes.53.8.2110. PMid:15277393.

72. Wayhs CA, Manfredini V, Sitta A, Deon M, Ribas GS, Vanzin CS, et al. Effects of insulin and clonazepam on DNA damage in diabetic rats submitted to the forced swimming test. Mutat Res. 2010;703(2):187-90. http://dx.doi. org/10.1016/j.mrgentox.2010.08.017. PMid:20816849. 
73. Wiernsperger NF. Oxidative stress as a therapeutic target in diabetes: revisiting the controversy. Diabetes Metab. 2003;29(6):579-85. http:// dx.doi.org/10.1016/S12623636(07)70072-1. PMid:14707886.

74. Zhao WQ, Alkon DL. Role of insulin and insulin receptor in learning and memory. Mol Cell Endocrinol. 2001;177(1-2):125-34. http://dx.doi. org/10.1016/S0303-7207(01)00455-5. PMid:11377828.

75. Guyot LL, Diaz FG, O'regan MH, Song D, Phillis JW. The effect of streptozotocin-induced diabetes on the release of excitotoxic and other amino acids from the ischemic rat cerebral cortex. Neurosurgery. 2001;48(2):385-90, discussion 390-1. PMid:11220383.

76. Kamboj SS, Sandhir R. Protective effect of $\mathrm{N}$-acetylcysteine supplementation on mitochondrial oxidative stress and mitochondrial enzymes in cerebral cortex of streptozotocin-treated diabetic rats. Mitochondrion. 2011;11(1):21422. http://dx.doi.org/10.1016/j. mito.2010.09.014. PMid:21059408.

77. Arnal E, Miranda M, Barcia J, BoschMorell F, Romero FJ. Lutein and docosahexaenoic acid prevent cortex lipid peroxidation in streptozotocininduced diabetic rat cerebral cortex. Neuroscience. 2010;166(1):2718. http://dx.doi.org/10.1016/j. neuroscience.2009.12.028. PMid:20036322.
78. Wang CF, Li DQ, Xue HY, Hu B. Oral supplementation of catalpol ameliorates diabetic encephalopathy in rats. Brain Res. 2010;1307:158-65. http://dx.doi. org/10.1016/j.brainres.2009.10.034. PMid:19852947.

79. Okouchi M, Okayama N, Aw TY. Preservation of cellular glutathione status and mitochondrial membrane potential by $\mathrm{N}$-acetylcysteine and insulin sensitizers prevent carbonyl stress-induced human brain endothelial cell apoptosis. Curr Neurovasc Res. 2009;6(4):267-78. http://dx.doi. org/10.2174/156720209789630348. PMid:19807652.

80. Liu J, Wang S, Feng L, Ma D, Fu $Q$, Song $Y$, et al. Hypoglycemic and antioxidant activities of paeonol and its beneficial effect on diabetic encephalopathy in streptozotocininduced diabetic rats. $J$ Med Food. 2013;16(7):577-86. http:// dx.doi.org/10.1089/jmf.2012.2654. PMid:23875897.

81. Lu M, Xu L, Li B, Zhang W, Zhang $\mathrm{C}$, Feng $\mathrm{H}$, et al. Protective effects of grape seed proanthocyanidin extracts on cerebral cortex of streptozotocin-induced diabetic rats through modulating AGEs/RAGE/ NF-kappaB pathway. J Nutr Sci Vitaminol (Tokyo). 2010;56(2):87-97. http://dx.doi.org/10.3177/jnsv.56.87. PMid:20495289.
82. Kuhad A, Chopra K. Curcumin attenuates diabetic encephalopathy in rats: behavioral and biochemical evidences. Eur J Pharmacol. 2007;576(1-3):34-42. http://dx.doi. org/10.1016/j.ejphar.2007.08.001. PMid:17822693.

83. Samarghandian S, Azimi-Nezhad M, Samini F. Ameliorative effect of saffron aqueous extract on hyperglycemia, hyperlipidemia, and oxidative stress on diabetic encephalopathy in streptozotocin induced experimental diabetes mellitus. Biomed Res Int. 2014;2014:920857. PMID: 25114929.

84. Xue HY, Jin L, Jin LJ, Li XY, Zhang P, Ma YS, et al. Aucubin prevents loss of hippocampal neurons and regulates antioxidative activity in diabetic encephalopathy rats. Phytother Res. 2009;23(7):980-6. http://dx.doi. org/10.1002/ptr.2734. PMid:19140154.

85. Xue H, Jin L, Jin L, Zhang P, Li D, Xia $Y$, et al. Neuroprotection of aucubin in primary diabetic encephalopathy. Sci China C Life Sci. 2008;51(6):495-502. http://dx.doi.org/10.1007/s11427-0080069-x. PMid:18488169.

86. Xue HY, Lu YN, Fang XM, Xu YP, Gao GZ, Jin LJ. Neuroprotective properties of aucubin in diabetic rats and diabetic encephalopathy rats. Mol Biol Rep. 2012;39(10):9311-8. http://dx.doi. org/10.1007/s11033-012-1730-9. PMid:22810648.

Recebido: Out 13, 2015 Aceito: Dez 01, 2015 